\title{
The management of the patient with headache: from evidence to clinical practice
}

\author{
Paola Gnerre, ${ }^{1}$ Ombretta Para, ${ }^{2}$ Francesco De Antoniis, ${ }^{3}$ Danilo Cavalieri, ${ }^{4}$ Marianna Piombo ${ }^{5}$ \\ ${ }^{1}$ Department of Internal Medicine, San Paolo Hospital, Savona; ${ }^{2}$ Department of Internal Medicine, AOU Careggi, Firenze; \\ ${ }^{3}$ Emergency Department, Misericordia e Dolce Hospital, Prato; ${ }^{4}$ Internal Medicine, Biomedical Campus, Roma; ${ }^{5}$ Neurology, \\ Biomedical Campus, Roma, Italy
}

\begin{abstract}
Headaches are the most prevalent neurological disorders and continue to be a frequent cause of Emergency Department use, accounting for $2 \%$ of all visits. Although it is a neurological disease it must be also a clinical competence of internist for its nature and frequency. The keys to effective management of these syndromes are differential diagnosis through history, physical examination, and diagnostic studies when necessary. This monograph highlights the importance of establishing whether headache is a secondary symptom of another disease process or a primary condition. The goal of headache management is for patients and healthcare professionals to work together to minimize pain and improve quality of life.
\end{abstract}

\section{Introduction}

Headache is a painful disorder of the head sometimes associated with pain of the face and/or neck. ${ }^{1}$ Headaches are the most prevalent neurological disorders and continue to be a frequent cause of Emergency Department (ED) use, accounting for $2 \%$ of all visits. In 1988 the International Headache Society (IHS) published a first classification system for headache disorders. Now a third edition is now to being final. ${ }^{2}$ This classification is hierarchical, and you must decide how detailed you want to make your diagnosis. This can range from the first-digit level to the fifth. First, one gets a rough idea about which group the patient belongs to. For this reason it could be adapted in different clinical setting. Moreover, this classification gives operative criteria for diagnosis, not only descriptions. This system is

Correspondence: Paola Gnerre, Department of Internal Medicine, San Paolo Hospital, via Genova I, 17100 Savona, Italy. Tel.: +39.0198404358 - Fax: +39.0198404583.

E-mail: pgnerre@yahoo.it

Key words: headache, evidence-based recommendations, clinical approach.

Received for publication: 4 October 2013.

Revision received: 9 January 2014.

Accepted for publication: 12 February 2014.

This work is licensed under a Creative Commons Attribution NonCommercial 3.0 License (CC BY-NC 3.0).

C Copyright P. Gnerre et al., 2015

Licensee PAGEPress, Italy

Italian Journal of Medicine 2015; 9:37-48

doi:10.4081/itjm.2015.436 not easily applicable in clinical practice but it is useful to divide headache into two categories: primary and secondary headache. Primary headaches (Table 1$)^{2}$ and its associated features occur in the absence of any exogenous cause. On the contrary secondary headaches recognize an exogenous cause as meningitis, intracranial, hemorrhage, brain tumor or the temporal arteritis. Although the majority of chronic pain cephalic-applicants are of benign origin, each case deserves a thorough study in the attempt to pursue and to establish the possible etiology for a correct prognostic and therapeutic diagnosis. ${ }^{3}$ The objective of this monograph is to provide evidencebased recommendations for the proper management of headache by analysis of the guidelines available to date.

\section{Epidemiology}

Headaches are the most prevalent neurological disorders and are among the most frequent symptoms seen in general practice. The headache is about $30 \%$ of neurological specialist visits and continues to be a frequent cause of ED use, accounting for $2 \%$ of all visits. ${ }^{4} 50 \%$ of the general population have headaches during any given year, and more than $90 \%$ of them report a lifetime history of headache. ${ }^{5}$ The only Italian epidemiological study (PACE study) ${ }^{6}$ showed that $52 \%$ of men a and $43 \%$ of women interviewed suffered from headache in the last year. ${ }^{7}$

\section{Primary headache}

\section{Migraine}

Introduction

Migraine is a common, debilitating headache disor- 
der, characterized by recurring attacks of severe headache and autonomic nervous system dysfunction, that may be preceded in some patients by transient focal neurologic symptoms known as aura. ${ }^{89}$ Migraine is the second most common headache disorder, after tensiontype headache, globally affecting about $11-12 \%$ of adult population. ${ }^{10,11}$ Prevalence is three times higher in women affected about $18 \%$ of the female population, and $6 \%$ of men. ${ }^{11}$ Migraine is common also in children; the prevalence is 3\% in children aged 3-7 years, $4 \%$ $11 \%$ in those $7-11$ years, and $8 \%-23 \%$ in teenagers. ${ }^{12}$ The mechanisms of migraine remain incompletely understood. Therefore migraine is believed to be a neurovascular disorder with evidence supporting its mechanisms starting within the brain and then spreading to the blood vessels. ${ }^{8}$ Migraine results in a marked decrease in the patient's quality of life: it is currently ranked by the World Health Organization (WHO) as $19^{\text {th }}$ among the causes of disability in one's life. ${ }^{13}$ Despite this, migraine remains often underdiagnosed and the available therapies underused, with a consequent increase of physical, emotional, social and economic burdens. ${ }^{15-17}$ On account of this, improving diagnosis and optimizing treatment could reduce substantially this global burden.

\section{Clinical manifestation}

Migraine can be divided into two major sub-types: migraine without aura (previously known as common migraine) and migraine with aura. Migraine is characterized by attacks of moderate or severe head pain, that may be bilateral or frequently unilateral, usually frontotemporal often throbbing and aggravated by routine activities of daily living. Nausea and vomiting usually occur late in the attack. Some patients have been noted to be pale and clammy, especially if nausea develops. Photophobia and/or phonophobia also are commonly associated with the headache. Lightheadedness is frequent. Specific features of the headache can distinguish migraine from other headache. When untreated these attacks typically last from 4 to $72 \mathrm{~h}$ and occur between once a week and once a year, average one per month, less than 15 days per month (episodic migraine) ${ }^{8,9}$ More frequent attacks, 15 or more days per month for more than 3 months, which has the features of migraine headache on at least 8 days per month codified the entity of chronic migraine. ${ }^{18}$ The most common cause of symptoms suggestive of chronic migraine is medication overuse. ${ }^{2}$ In one third of patients the migraine attacks of headache are usually preceded or accompanied or sometimes substituted by transient focal neurologic symptoms, termed auras. The diagnosis for these patients is migraine with aura. ${ }^{19,20}$ Depending on clinical presentation of headache, migraine with aura is divided into typical aura with headache and typical aura without headache. Auras consist most commonly of visual symptoms (hemianopic disturbance or a spreading scintillating scotoma) and less commonly of sensory symp-

Table 1. Primary headache.

\begin{tabular}{|c|c|}
\hline Migraine & $\begin{array}{l}\text { Migraine with aura } \\
\text { Migraine without aura } \\
\text { Migraine with brainstem aura } \\
\text { Hemiplegic migraine } \\
\text { Chronic migraine } \\
\text { Complications of migraine } \\
\text { Probable migraine } \\
\text { Episodic syndromes that may be associated with migraine }\end{array}$ \\
\hline Tension-type headache & $\begin{array}{l}\text { Infrequent episodic tension-type headache } \\
\text { Frequent episodic tension-type headache } \\
\text { Chronic tension-type headache } \\
\text { Probable tension-type headache }\end{array}$ \\
\hline Trigeminal autonomic cephalalgias & $\begin{array}{l}\text { Cluster headache } \\
\text { Paroxysmal hemicrania } \\
\text { Short-lasting unilateral neuralgiform headache attacks with conjunctival injection and tearing } \\
\text { Short-lasting unilateral neuralgiform headache attacks with cranial autonomic symptoms } \\
\text { Hemicrania continua } \\
\text { Probable trigeminal autonomic cephalalgia }\end{array}$ \\
\hline Other primary headache disorders & $\begin{array}{l}\text { Primary exercise headache } \\
\text { Primary cough headache } \\
\text { Primary headache associated with sexual activity } \\
\text { Primary thunderclap headache } \\
\text { Primary stabbing headache } \\
\text { Hypnic headache } \\
\text { Cold-stimulus headache } \\
\text { External-pressure headache } \\
\text { New daily persistent headache }\end{array}$ \\
\hline
\end{tabular}

Modified from Headache Classification Committee of the International Headache Society, 2013. ${ }^{2}$ 
toms such as unilateral hypoesthesia or paresthesia of hand, arm or face or disturbance of speech (dysphasic speech disturbance) or motor symptoms. Aura usually develops gradually over 5-20 min and lasts no longer than $60 \mathrm{~min}$. It is characterized by a mix of positive and negative features and is complete reversible. ${ }^{8,9}$ Aura of longer duration that could be a possible complication, is rare and justifies further investigations. A form of aura characterized by symptoms as dysarthria, vertigo, tinnitus, hypoacusia, diplopia, ataxia, decreased level of consciousness, simultaneous bilateral paresthesias without motor symptoms, associated or followed by migraine is typical of a distinct subtype of migraine called migraine with brainstem aura previously called basilar artery migraine. These symptoms clearly originate from brainstem and/or from bilateral cerebral hemisphere. ${ }^{89}$ Basilar-type attacks are mostly seen in young adults. Many patients who have basilar-type attacks also repot attacks with typical aura. Migraine with aura including motor weakness is called hemiplegic migraine. The presence of migraine with aura including motor weakness identifies familial hemiplegic migraine: if this is present in at least one first or second-degree relative, it identifies familial hemiplegic migraine. For complications of migraine ICD-III codifies four entities: i) status migrainosus: a debilitating migraine attack lasting for more than $72 \mathrm{~h}$; ii) persistent aura without infarction: aura symptoms persisting for 1 week or more without evidence of infarction on neuroimaging; iii) $\mathrm{mi}$ grainous infarction: evidence of an ischemic brain lesion demonstrated by neuroimaging; iv) and migraine aura-triggered seizure: a seizure triggered by an attack of migraine with aura. Recurrent episodic attacks, usually stereotypical, of vomiting and intense nausea associated with pallor and lethargy (cyclical vomiting) or attacks of episodic midline abdominal pain associated with vasomotor symptoms (abdominal migraine) or recurrent brief episodic attacks of vertigo (benign paroxysmal vertigo of childhood) could be explained as cyclical vomiting syndromes commonly precursors to migraine. ${ }^{8,9}$ Finally the ICHD-III provides for the definition of probable migraine. This entity is a migrainous disorder in which attacks and/or headache missing one of the features needed to fulfill all criteria for a disorder coded. The ICHD-III divides these into two categories: probable migraine without aura and probable migraine with aura.

A variety of external and internal factors have been demonstrated to precipitate migraine attacks. Several studies demonstrated that stress, lack of sleep, hormonal factors in women, perfume or odor, alcohol intake, smoke, excessive caffeine or specific food (as chocolate) consumption and fasting are the most common trigger factors. ${ }^{21}$ The guidelines recommend to consider neuroimaging in migrainers with an unexplained abnormal finding on neurologic examination and in patients with atypical headache features of migraine, whereas neuroimaging generally is not indicated for patients with migraine and a normal neurologic examination.

\section{Therapy}

In view of marked decrease in the quality of life of the patient with migraine it is important to optimize treatment. A comprehensive treatment approach to migraine may include as a first step non-pharmacologic measures, as well as abortive and prophylactic medications. Non-pharmacologic therapies include education of the patient about the disorder and its mechanisms and changes in lifestyle. Thus, regular sleep, regular meals, exercise, avoidance of peaks of stress and troughs of relaxation, and avoidance of dietary triggers can be helpful. The crucial message is that patient should aim for a regularity of habits. ${ }^{22,23}$ Regarding pharmacologic therapies, drugs for the treatment of migraine can be divided into prophylactic medications, that are taken daily whether or not headache is present to reduce the frequency and severity of attacks (propranolol, metoprolol, amitriptyline, flunarizine, valproate, topiramate) ${ }^{23,24}$ and abortive drugs, that are taken to treat attacks as they arise. Treatments for attacks can be further divided into nonspecific (aspirin, acetaminophen, non-steroidal antiinflammatory drugs, $)^{23,25}$ and migraine-specific treatments (ergotamine, dihydroergotamine, triptans). ${ }^{23,26}$ Therefore triptans are the first choice when a specific treatment is needed.

\section{Tension-type headache}

\section{Introduction}

Tension-type headache (TTH) is a neurological disorder characterized by attacks of mild or moderate head pain. ${ }^{27}$ TTH is the most common cause of headache in the general population and is usually selfmanaged. The rate of episodic tension-type headache ranges from 12 to $78 \%{ }^{28}$ The peak prevalence in both sexes is at 40-49 years but children are often affected. ${ }^{29}$ The chronic tension-type headache is less common, affecting about $3 \%$ of the general population. ${ }^{30}$ Furthermore the prevalence of episodic TTH increases with the level of education. ${ }^{31}$ The underlying cause of tension-type headache is unknown. The most likely explanation for the sporadic type is to be found in a hyper excitable of sensory neurons from peripheral afferent muscles of the head and neck. Muscle tenderness and psychological tension are probably associated with infrequent tension-type headache. The alterations of the central processes of pain are present in some patients with chronic tension-type headache. ${ }^{32}$

\section{Clinical manifestation}

Patients usually report a pain present on both side of the forehead, temples or back of the head as a band 
from mild to moderate intensity that may be bilateral and pressing or tightening but non pulsating and that is not aggravated by routine activities. This type of headache may last from hours to days and is not associated with constitutional or neurological symptoms. Unlike migraine, there is no nausea or vomiting. However, like migraine, either photophobia (discomfort from light) or phono-phobia (discomfort from sound) may be present. Tension-type headache has no prodrome (symptoms indicating onset of headache) or aura (such as flashing lights). ${ }^{33}$ The diagnosis is based on history and neurological examination. Rarely require imaging studies of the head such as magnetic resonance imgaing (MRI). TTH is divided into episodic and chronic depending on the frequency of symptoms. The episodic form occurs fewer than 15 days per month. The chronic form occurs 15 or more days per month. The episodic tension-type headache is divided into two subtypes: infrequent and frequent. The infrequent episodic tension-type headache has very low impact on daily life and occurs $<1$ day a month $(<12$ days a year on average). The frequent episodic tension-type headache occurs $>1$ and $<15$ days a month $(>12$ and $<180$ days a year). The episodic tension-type headache is divided into two subtypes: infrequent and frequent. The infrequent episodic tension-type headache has very low impact on daily life and occurs $<1$ day a month $(<12$ days a year on average). The frequent episodic tension-type headache occurs $>1$ and $<15$ days a month ( $>12$ and $<180$ days a year) ${ }^{34}$ Chronic tension-type headache evolves over time from frequent episodic tension-type headache. When headache that fulfilled the criteria for TTH is daily and unremitting from less than $24 \mathrm{~h}$ after its first onset ICHD-III codifies new daily persistent headache While episodic form is seldom severe or disabling and generally it does not interfere with normal quality of life and work, the chronic form is an important disease which causes decrease in the quality of life, significant disability and it has high social and economic costs. At last probable tension-type headache is the form of TTH that not fulfills standard criteria.

\section{Therapy}

The main preventive measure for TTH is avoiding exposure to situations that trigger headache. Treatment may include non-steroidal anti-inflammatory drugs (such as ibuprofen) and analgesics (such as acetaminophen), behavioral therapies (such as meditation, biofeedback, or yoga), improvement of posture, and stretching/relaxation exercises. ${ }^{32}$

\section{Trigeminal autonomic cephalalgias}

\section{Introduction}

Trigeminal autonomic cephalalgias (TACs) are a group of primary headache that are characterized by attacks of severe unilateral pain in a trigeminal distribution. TACs includes cluster headache, paroxysmal hemicranias $(\mathrm{PH})$, short-lasting unilateral neuralgiform headache attacks with conjunctival injection and tearing (SUNCT), short-lasting unilateral neuralgiform headache attacks with cranial autonomic symptoms (SUNA), hemicrania continua (HC) and probable trigeminal autonomic cephalalgia. ${ }^{9}$ They are distinguished (Table 2$)^{35}$ by the frequency of attacks of pain, the length of the attacks and very characteristic response to medical therapy, such that the diagnosis can usually be made clinically, which is important because it dictates therapy.

\section{Clinical manifestation}

Cluster is the most common TACs. Onset of cluster headache typically occurs at 20-40 years of age and the condition has a prevalence of $80-100$ per 100,000 persons. ${ }^{36}$ Men have cluster headache five to seven times more frequently than women. Although the prevalence in the general population is much lower than migraine and tension-type headache, cluster

Table 2. Clinical features of the trigeminal autonomic cephalalgias.

\begin{tabular}{llll}
\hline & Cluster headache & Paroxysmal hemicrania & SUNCT \\
\hline Type of pain & Stabbing, boring & Throbbing, boring, stabbing & Burning, stabbing, sharp \\
\hline Severity & Excruciating & Excruciating & Excruciating \\
\hline Site & Orbit, temple & Orbit, temple & Periorbital \\
\hline Duration attacks & $15-180$ min & $2-30$ min & $5-240 \mathrm{~s}$ \\
\hline Attack frequent & $1 /$ alternate day-8 day & $\begin{array}{l}1-40 / \text { days }(>5 / \text { days for more } \\
\text { than half the time })\end{array}$ & $3-200 /$ days \\
\hline Abortive treatment & $\begin{array}{l}\text { Sumatriptan injection or nasal spray } \\
\text { Oxygen }\end{array}$ & Nil & Nil \\
\hline Prophylactic treatment & Verapamil, lithium, topiramate & Indometacin & Lamotrigine, topimarate, gabapentil \\
\hline
\end{tabular}

SUNCT, short-lasting unilateral neuralgiform headache attacks with conjunctival injection and tearing. Modified from Lambru et al., 2012.35 
headache is a form of headache very relevant for the suffering of the patient with female patients describing each attacks as being worse than childbirth. ${ }^{35}$ The mechanism behind cluster headache is largely unknown. It is possible that cluster headache is partially caused by disturbances in the hypothalamus including secondary activation of pain-sensitive nerves. Clinical features make easily recognized. Cluster headaches are severe, unilateral headaches with burning and drilling pain around or above one eye or temporally. ${ }^{2}$ In the majority of cases, the same side is affected in all attacks but the pain can change sides. The pain typically has a duration of 45-90 (15-180) min and may occur several times a day. In some patients, the attacks occur at roughly the same time of the day every day, and in other clusters occur in the same period every year. ${ }^{37}$ Permanent remission is seen, but after 15 years with the disease, $80 \%$ of patients still have attacks. However, about $10-15 \%$ of patients have chronic symptoms without remissions. The attacks are associated with one or more of the following autonomic symptoms, all of which are ipsilateral: conjunctival injection, lacrimation, nasal congestion, rhinorrhea, forehead and facial sweating, miosis, ptosis and edema of the eyelid as signs of parasympathetic hyperactivity and sympathetic hypo-activity. Patients are typically restless and agitated during attacks. Attacks often occur at night, typically 1-2 $\mathrm{h}$ after the patient has fallen asleep. Migraine-like symptoms such as nausea, photophobia and phono-phobia may also occur. ${ }^{38}$ The IHS classifies ${ }^{9}$ the cluster headache in episodic and chronic. The most common form of cluster headache is episodic (80-90\%): the intervals of remission between a phase of cluster and the following may be a few months or, in the most benign form, of several years. Instead in the chronic cluster headache (present in about $10 \%$ of cases), the attacks are repeated several times a day without outages with remissions of less than one month. Some patients may switch from chronic to episodic cluster headache. Indeed the paroxysmal hemicranias are characterized by attacks with similar characteristics of pain and associated symptoms and signs to those of cluster headache, but they are shorter-lasting $(2-30 \mathrm{~min})$, more frequent (more of 5/day) and they occur more commonly in females and respond absolutely to indomethacin. The SUNCT are characterized by short-lasting attacks of unilateral pain and they are every so often accompanied by prominent lacrimation and redness of the ipsilateral eye. The frequency of attacks is between 3 and 200 attacks for day. In SUNA, there may be cranial autonomic symptoms other than conjunctival injection and lacrimation. ${ }^{38}$ However, SUNA still needs to be properly validated. $\mathrm{HC}$ is a strictly unilateral, continuous, moderate to severe headache that fluctuates in intensity with possible exacerbations of severe pain associated with autonomic disturbances. It is absolutely responsive to indomethacin. $\mathrm{HC}$ has been included under this group in ICHD-3 on the basis that the pain is typically unilateral as are the cranial autonomic symptoms when present. ${ }^{2}$

\section{Therapy}

Subcutaneous injection of $6 \mathrm{mg}$ sumatriptan is recommended as the first choice treatment for the relief of acute attacks of cluster headache while nasal sumatriptan or zolmitriptan and $100 \%$ oxygen (7-12 L per min) are recommended for treatment of acute attacks of cluster headache in patients who cannot tolerate subcutaneous sumatriptan. For the prophylaxis of cluster headache is recommended verapamil 240-960 $\mathrm{mg}$ while indomethacin up to $225 \mathrm{mg}$ is recommended for the prophylaxis of paroxysmal hemicranias. Indometacin is the treatment of choice of PH. Lidocaine is effective in some cases of SUNCT/SUNA, headaches otherwise difficult to treat. ${ }^{38}$

\section{Other headache}

This chapter includes headaches that are clinically heterogeneous. The pathogenesis of these types of headache is still poorly understood, and their treatment is suggested on the basis of anecdotal reports or uncontrolled trials. These are forms which occur in predisposed individuals only after exposure to particular situations, such as headaches from coughing, stress, physical activity, or sexual activity. All of the syndromes mentioned below require a differential diagnosis with a secondary headache. ${ }^{9}$ Primary stabbing headach $e^{39}$ is characterized by transient and localized stabs of pain in the head that occur spontaneously in the absence of organic disease of underlying structures or of the cranial nerves. In a single published descriptive study, $80 \%$ of stabs lasted 3 seconds or less. Stabbing pains are more commonly experienced by people subject to migraine (about $40 \%$ ) or cluster headache (about $30 \%$ ). A positive response to indomethacin has been reported in some uncontrolled studies. Primary cough headache is headache precipitated by coughing or straining in the absence of any intracranial disorder. It is usually bilateral and it affects predominantly patients older than 40 years of age. Indomethacin is usually effective in the treatment of primary cough headache. ${ }^{40}$ Primary exertion headache is a headache precipitated by any form of exercise. This headache occurs particularly in hot weather or at high altitude. Indomethacin has been found effective in the majority of the cases. Primary headache associated with sexual activity is a headache precipitated by sexual activity, usually starting as a dull bilateral ache as sexual excitement increases and suddenly becoming intense at orgasm, in the absence of any intracranial disorder. ${ }^{40}$ It can be divided into: pre-orgasmic headache and or- 
gasmic headache. Postural headache resembling that of low cerebrospinal fluid pressure has been reported to develop after coitus. An association between primary exertional headache and migraine is reported in approximately $50 \%$ of cases. Hypnic headache, previously termed alarm clock is characterized by attacks of dull headache that always waken patient from sleep. The pain is usually mild to moderate, but severe pain is reported by approximately $20 \%$ of patients. ${ }^{40}$ Pain is bilateral in about two-thirds of cases. The attack usually lasts from 15 to $180 \mathrm{~min}$, but longer durations have been described. Caffeine and lithium have been effective treatments in several reported cases. New daily persistent headache indicates persistent headache daily from its onset, which is clearly remembered. The pain may be migraine-like or tension-type like or have elements of both. ${ }^{2}$

\section{Medication overuse headache}

When a headache occurs for the first time in close temporal relation to exposure to or withdrawal from a substance, it is coded as a secondary headache attributed to exposure to or withdrawal from that substance. ${ }^{2}$ Medication overuse headache is a headache occurring on 15 or more days per month developing as a consequence of regular overuse of acute or symptomatic headache medication (on 10 or more, or 15 or more days per month, depending on the medication) for more than 3 months. ${ }^{2}$ Overuse of any class of drugs as triptans, ergots, opioids or combination analgesic useful for the treatment of acute headaches can lead to the development of this entity. Medication overuse headache must be excluded in all patients with chronic daily headache (headache $\geq 15$ days/month for $>3$ months) ${ }^{41}$ Treatment of medication overuse headache requires withdrawal as an initial step, initiation of preventive treatment and education of patient.

\section{Thunderclap headache}

The thunderclap headache (TCH) has a sudden onset reaching its own peak in few seconds or minutes as a thunder occurring in the clear sky. ${ }^{42} \mathrm{The} \mathrm{TCH}$ estimated incidence in the West Countries is 43 cases for 100,000 adults..$^{43} \mathrm{TCH}$ represents one of the most recurring symptom of neurological disease in the ED. ${ }^{44}$ The first etiology to be searched in case of a suddenonset headache is properly represented by the subarachnoid hemorrhage (ESA). ${ }^{45}$ In fact many systematic reviews and observational studies have demonstrated that about $70 \%$ of ESA is characterized by a thunderclap pattern. ${ }^{46}$ However ESA is misidentified in $1 / 3$ of patients, in particular in those that have only headache and have a normal Glasgow coma score. ESA is caused in $85 \%$ of cases by the breakage of a cerebral artery aneurysm. It has to be borne in mind that ESA stands for $11-25 \%$ of all TCH causes. ${ }^{9}$ And then it is necessary to consider other suitable etiologies, related to the vascular origin such as cerebral venous sinus thrombosis, cervical artery dissection, ischemic stroke, hypophysis apoplexia, reversible cerebral vasoconstriction syndrome, reversible posterior leukoencephalopathy. The non-vascular etiology consists of the cerebral hypotension and the colloid cyst of the third ventricle. ${ }^{42}$ Primary thunderclap is diagnosed when no underlying cause is discovered. Secondary headaches are of important consideration, not only because of their potential danger, but also because of their diffusion. Whereas there is a lack of robust knowledge about the prevalence of the secondary headaches, there are quite good epidemiological data on some of the more well-defined underlying disorders, and there are also several patient-based studies reporting the proportion with the underlying disorder that will develop headache. For example $90 \%$ of patients with temporal arteritis and with subarachnoid hemorrhage have headache. ${ }^{46}$ Clinical manifestation can be completely unspecific and may be similar to clinical presentation of migraine or tension-type headache. Red flags for secondary disorders ${ }^{1}$ as reported in Table $3^{1}$ include new or change in headache after 50 years of age, increased frequency or severity of headache, new onset of headache with an underlying medical condition, headache with concomitant systemic illness, focal neurologic signs or symptoms, papilledema and headache subsequent to head trauma. A neurologic examination must be performed and abnormal findings should be carried further diagnosis. The earliest step is to execute a cerebral-computed tomography (CT) without contrast. If the $\mathrm{CT}$ will be negative for expansive process and intracranial hypertension signs it is necessary made a lumbar puncture. ${ }^{47}$ If the cerebrospinal fluid (CSF) has blood spots, it should be immediately centrifuged. A yellow color supernate, known as xantocromia, is suggesting a subarachnoid hemorrhage. If CSF is clear, it is useful to measure the opening pressure (CSF pressure). High CSF pressure could suggest cerebral venous thrombosis or intracranial hypertension while a low CSF pressure could specify intracranial hypotension. ${ }^{47}$ Negative CT does not keep out the subarachnoid hemorrhage. $\mathrm{CT}$ is negative for $2 \%$ of cases in the first $12 \mathrm{~h}$ from the onset; in addition it is negative for $10 \%$ of cases in the first $24 \mathrm{~h}$ and for $50 \%$ of cases in the next 5 days. Systematic reviews have suggested that MRI sensitivity is like to that of CT in the first hours, but it would become more sensitive after the first day. However, MRI sensitivity in the earliest phases is not of $100 \%$. Further steps to unveil the TH main cause consist of CT angiography, MR angiography and traditional percutaneous angiography. ${ }^{42}$ 
The management of the patient with headache: rationale and objective

Headache is the most common pain syndrome and it is among the most common reasons for patients to seek medical attention. For these reasons the diagnosis and management of headache should be a competence of internist. It is not the subject of this work headache in children and adolescents. Therefore, the goal of this work is to raise awareness of the clinical management of headache through a better knowledge of its diagnosis and its treatment.

\section{The management of headache: methodology}

In order to provide evidence-based recommendations for the management of patients with headache, we first verified the existence of guidelines on the matter. Therefore, we conducted a search using the following database guidelines:

- Scottish Intercollegiate Guidelines Network (SIGN);

- Institute for Clinical Systems Improvement (ICSI);

- National Istitute for Health and Care Excellence

(NICE) (NHS evidence);

- National Guideline Clearinghouse;

- Canadian Medical Association (CMA Infobase);

- New Zealand Guidelines Group;

- National System Guidelines;

- Clinical Practice Guidelines Portal;

- EGuidelines.
The research was carried out by five authors independently, using as key-words terms headache, migraine, headache tension-type and cluster when the site included the search function, and in other cases we listed the last manually guidelines stored in the database or made reference to the neurological illness. The results obtained separately were then compared and discussed together. The guidelines thus obtained were evaluated using the AGREE instrument (Appraisal of Guidelines, Research and Evaluation II, 22) ${ }^{48}$ by 5 authors independently, in order to identify the guidelines qualitatively better. AGREE II assesses compliance with 23 requirements, meeting 6 domains as the explanation of the purpose, the clarity, the involvement of all stakeholders, the rigor of development, applicability and editorial independence of the same. Each author assessed the compliance of individual requirements with a score from 1 (disagree completely) to 7 (complete agreement). The scores assigned by each author were added within individual domains and reported with the highest and the lowest score possible within the domain based on the number of requirements included and the number of evaluators.

\section{The management of headache: results}

Through the databases listed above, we identified 82 guidelines: 69 were excluded based on the title or the text or for other reasons and 13 were selected for evaluation. Of these, eight evaluate management of all

Table 3. Red flag features.

New onset or change in headache in patient who are aged over 50 years old

Thunderclap: rapid time to peak headache intensity (seconds to $5 \mathrm{~min}$ )

Focal neurological symptoms (e.g. limb weakness, aura $<5$ min or $1 \mathrm{~h}$ )

Non-focal neurological symptoms (e.g. cognitive disturbance)

Change in headache frequency, characteristics or associated symptoms

Abnormal neurological examination

Headache that change with posture

Headache weakening the patient up (migraine is the most frequent cause of morning headache)

Headache precipitated by physical exertion or Valsalva maneuver (e.g. coughing, laughing, straining)

Patients with risk factors for cerebral venous sinus thrombosis

Jaw claudication or visual disturbance

Neck stiffness

Never

New onset headache in a patient with a history of human immunodeficiency virus

Infection

New onset headache in a patient with a history of cancer

Modified from Clinch, 2001.' 
headaches, four assess the management of migraine, one assesses the management of trigeminal autonomic cephalalgias. The overall quality of selected guidelines was assessed by 5 authors using the AGREE instrument II. The classification of the IHS is a classification system globally accepted for headache disorders but it is not easily applicable in clinical practice. In view of these considerations and on the basis of the assessment of guidelines within the AGREE instrument, the guidelines produced by Scottish Intercollegiate Guidelines ${ }^{34}$ are qualitatively the best and whose implementation in clinical practice appears desirable. The Canadian guideline for migraine ${ }^{49}$ is of excellent quality (score 7), well written and full of information about the studies on various therapies for migraine prophylaxis and careful also to give appropriate advice for choosing between them. Sometimes, however, is a bit too specific. The Guideline for Primary Care Management of Headache in Adults (TOP and IHE) ${ }^{50}$ although not of high quality (score 5.5) due to lack of information about certain dimensions is of good quality, pretty comprehensive (dealing with all the headaches and not only one of the types and specifying for each is possible therapies that attack the prophylactic) and immediate. The recommendations are also well summarized in appropriate schemes and algorithms. The Evidence-Based Guidelines for Migraine Headache in the Primary Care Setting: Pharmacological Management for Prevention of Migraine (US Headache Consortium) ${ }^{51}$ is moderate quality (score 4 ). In fact, although it has been done a good job of reviewing the literature are not specified the methods of analysis and choice of the recommendations and does not provide specific guidance on the choice of drug therapies, but only a broad description of the same. The guideline of the National Guidelines of Clearinghouse ${ }^{52}$ is of good quality (score 6) and it is shown that there was clear ex- pression of the goals and motivations, good methodological rigor and discreet application. The NICE guideline ${ }^{53}$ (score 5.5) has discrete requirements of applicability but with less clarity. The European Federation of Neurological Societies (EFNS) guideline ${ }^{54}$ for the treatment of cluster headache and other trigeminal autonomic cephalalgia is of reasonable quality (score 5) although only focused on one type of primitive headache. The national guidelines made by the Italian Agency for the National Health Services (Age.Na.S) ${ }^{6}$ are guidelines for good quality (score 7), easily applicable but the writing in the Italian language restricts the diffusion.

\section{Clinical approach to patients with headache}

The management of headache consisted of five steps: i) evaluate the type of headache; ii) take a detailed history and assess functional impairment; iii) rule out cases for concern; iv) consider secondary headache disorder; v) refer to specialist when indicated. The diagnostic algorithm shown in Figure 1 can help us to better handle the patient with headache. ${ }^{9}$

\section{Evaluate the type of headache}

The first step in evaluating a patient with headache is to assess the type of headache based on the criteria established by the IHS. The patient should be asked to describe symptoms before and during the headache (Table 4). ${ }^{1}$ It is also important to determine the location (bilateral or unilateral), the quality (throbbing, stabbing, dull, pressure-like) and the intensity of the pain (mild or moderate or severe or very severe). Also an accurate assessment of the type of headache can be excluded causes of concern. Physicians should consider using headache diaries and appropriate assessment questionnaires to support the diagnosis and

Table 4. Questions for headache history.

Is this your first or worst headache?

How do you evaluate your headache on a scale from 1 to 10 ?

Do you have headache regularly?

What symptoms do you have before the headache starts?

What symptoms do you have during the headache?

When did this headache begin?

How did it start (gradually, suddenly, other)?

Where is your pain?

What kind of pain do you have (throbbing, stabbing dull or other)?

Do you have other medical problems?

Have you recently hurt your head or had a medical or dental procedure?

Do you take any medicine?

Modified from Clinch, 2001. 
management of headache. It is important to note that an inadequate history is the probable cause of most misdiagnosis of the headache type. ${ }^{1}$

\section{Take a detailed history and assess functional impairment}

The patient's history is of prime importance in the evaluation of the type of headache. The patient should be requested some questions about headache history
(Table 4). Physician should assess the presence of concurrent medical conditions. An organic cause of headache is more likely in patients with chronic medical condition, cancer, HIV and with acute viral syndrome or an acute bacterial infection. A complete examination is an essential first step in the evaluation. The general physical examination should include vital signs, fundoscopy and cardiovascular assessment, pal-

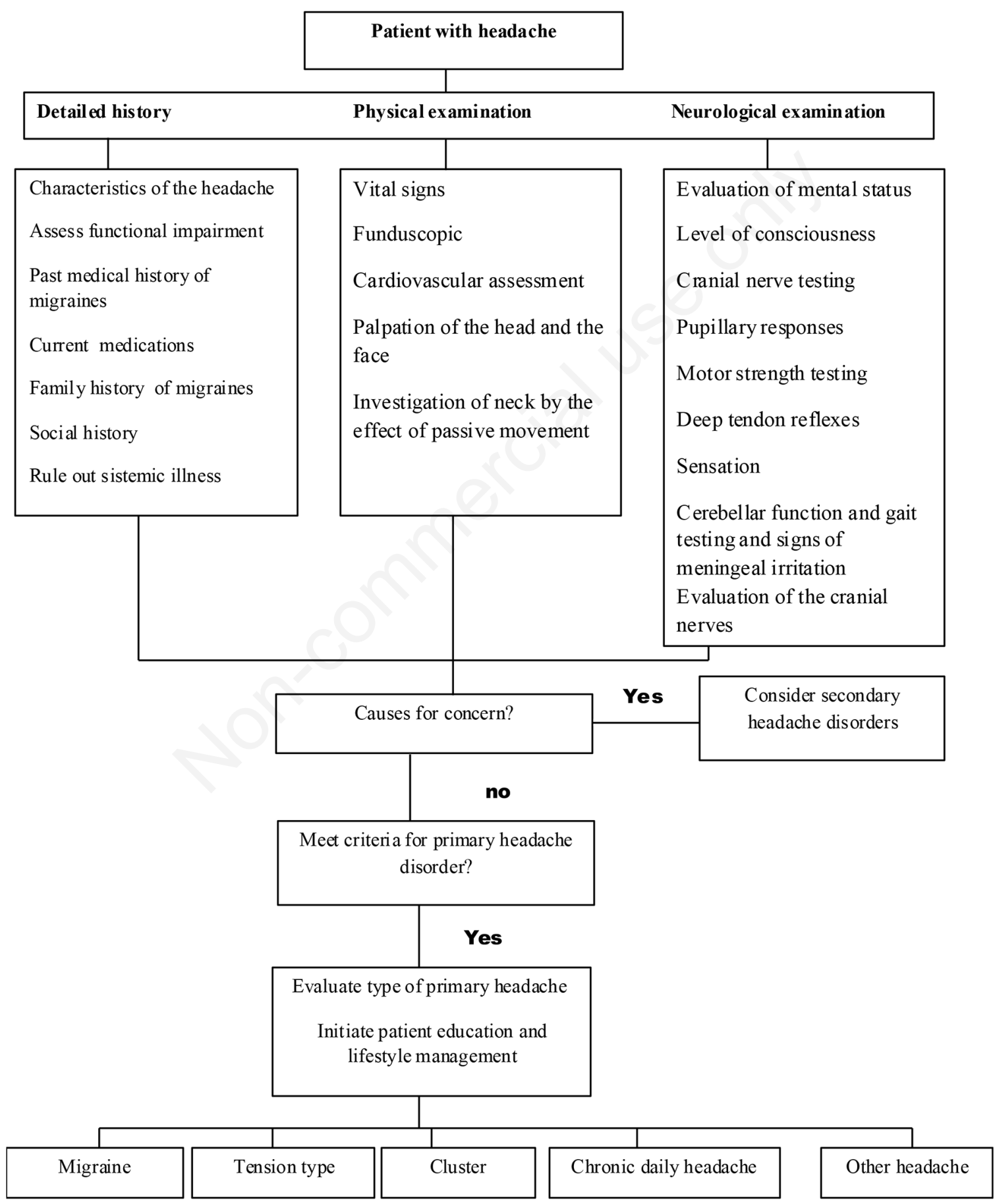

Figure 1. Diagnostic algorithm of headache. Modified from Stovner et al., 2007. ${ }^{9}$ 
pation of the head and the face and the investigation of neck by the effect of passive movement. The neurological evaluation should include mental status, level of consciousness, cranial nerve testing, pupillary responses, motor strength testing, deep tendon reflexes, pathologic reflexes, sensation, cerebellar function and gait testing and signs of meningeal irritation. ${ }^{1}$ A normal neurological examination without red flag features does not require a neuroimaging study.

\section{Rule out cases of concern}

The patient who presents with a new, severe headache is quite different from the patient with recurrent headaches over many years. In new-onset and severe headache, the probability of finding a potentially serious cause is considerably greater than in recurrent headache. If the headache is the first or worst headache you should consider carrying out further investigations. In fact patient with recent onset of pain require rapid diagnosis and management. You should think a serious disease when the headache is the first or worst, when abnormal neurological examination or fever are present, when vomiting precedes headache, when the pain is induced by bending, lifting or cough, when the pain disturbs sleep or is presented immediately upon awakening, when the headache onset after age 55 or is associated with local tenderness in region of temporal artery.

\section{Consider secondary headache disorder}

One of the greatest challenges within the field of headache is the identification of secondary headache disorders. Unfortunately, the signs and symptoms of secondary headache disorders can sometimes be subtle. Physicians must ask specific questions to identify red flags of secondary headache disorders (Table 3), conduct a detailed neurological examination and perform appropriate diagnostic testing in order to establish a diagnosis. A secondary headache disorders may be identified with the presence of diplopia, stiff neck, rash, fever, eye pain, unilateral paresthesias, disorientation and balance change. ${ }^{46}$ Differential diagnosis includes meningitis, subarachnoid hemorrhage, epidural or subdural hematoma, glaucoma, tumor, and purulent sinusitis. Finally it is important to assess any recent head trauma or dental procedure as well as any medications taken by the patient (especially caffeine-containing analgesics). Patients with red flag features should be evaluated by a CT or MRI study. ${ }^{45}$ Patients with thunderclap headache and a normal CT should have a lumbar puncture. Lumbar puncture should be delayed till $12 \mathrm{~h}$ after headache onset. In general emergent neurological imaging is recommended for a patient presenting with thunderclap headache with abnormal findings on neurological examination. Neuroimaging is also recommended to determine the safety of lumbar puncture in patient with headache, fever or nuchal rigidity accompanied by signs of increased intracranial pressure. Headache features that often warrant neuroimaging includes: isolated thunderclap headache, headache radiating to the neck, temporal headache in an older individual, headaches with increasing frequency or severity and headaches not responding to the treatment. ${ }^{46}$ New headache in HIV positive, in pregnancy, in patient older than 50 years of age or with a history of cancer required further investigations. Abnormality of neurological examination is the most predictor of intracranial pathologic conditions on subsequent imaging.

\section{Refer to specialist when indicated}

Specialty consultation may be considered when the diagnosis cannot be confirmed, etiology cannot be diagnosed or warning signals are present and headache occurs with a frequency or duration sufficiently to impair the quality of life of the patient despite to treatment. Failures in the treatment of headache are caused mainly by the fact that the doctors are not able to set the appropriate diagnosis. We need to keep in mind that often the patient with persistent headache suffers due to an incorrect diagnosis. ${ }^{9}$

\section{Conclusions}

Headache is one of the most common pain disorders and although it is a neurological disease it must be also a clinical competence of internist for its nature and frequency. The key to effective management of these syndromes is a differential diagnosis through history, physical examination, and diagnostic studies when necessary. This monograph highlights the importance of establishing whether headache is a secondary symptom of another disease process or a primary condition. The goal of headache management is for patients and healthcare professionals to work together to minimize pain and improve the quality of life.

\section{References}

1. Clinch CR. Evaluation of acute headaches in adults. Am Fam Physician 2001;63:685-92.

2. Headache Classification Committee of the International Headache Society (IHS). The International Classification of Headache Disorders, 3rd edition (beta version). Cephalalgia 2013;3:629-808.

3. De Luca GC, Bartleson JD. When and how to investigate the patient with headache. Semin Neurol 2010; 30:131-44.

4. Friedman BW, Grosberg BM. Diagnosis and management of the primary headache disorders in the emergency department of setting. Emerg Med Clin North Am 2009;27:71.

5. Stovner LJ, Andree C. Prevalence of headache in Eu- 
rope: a review for the Eurolight project. J Headache Pain 2010;11:289-99.

6. Age.Na.S. Linee guida nazionali di riferimento per la prevenzione e la terapia; settembre 2011. Available from: http://www.agenas.it

7. Mennini FS, Gitto L, Martelletti P. Improving care through health economics analyses: cost of illness and headache. J Headache Pain 2008;9:199-206.

8. Institute for Clinical Systems Improvement (ICSI). Health Care Guidelines: diagnosis and treatment of headache. 10th ed; January 2011. Available from: https://www.icsi.org/

9. Stovner LJ, Hagen K, Jensen R, et al. The global burden of headache: a documentation of headache prevalence and disability worldwide. Cephalalgia 2007;27:193-210.

10. Bigal ME, Lipton RB. The epidemiology, burden, and comorbidities of migraine. Neurol Clin 2009;27:321-34.

11. Lipton RB, Bigal ME. The epidemiology of migraine. Am J Med 2005;118:3S-10S.

12. Al-Twaijri WA, Shevell MI. Pediatric migraine equivalents: occurrence and clinical features in practice. Pediatr Neurol 2002;26:365-8.

13. World Health Organization. Headache disorders. Fact sheet No. 277; October 2012. Available from: http:/www.who.int/mediacentre/factsheets/fs277/en/

14. Lipton RB, Diamond S, Reed M, et al. Migraine diagnosis and treatment: results from the American Migraine Study II. Headache 2001;41:638-45.

15. Berg J, Stovner LJ. Cost of migraine and other headaches in Europe. Eur J Neurol 2005;12:59-62.

16. Cevoli S, D'Amico D, Martelletti P, et al. Underdiagnosis and undertreatment of migraine in Italy: a survey of patients attending for the first time 10 headache centres. Cephalalgia 2009;29:1285-93.

17. Leonardi M, Steiner TJ, Scher AT, Lipton RB. The global burden of migraine: measuring disability in headache disorders with WHO's Classification of Functioning, Disability and Health (ICF). J Headache Pain 2005;6:429-40.

18. Katsarava Z, Buse DC, Manack AN, Lipton RB. Defining the differences between episodic migraine and chronic migraine. Curr Pain Headache Rep 2012;16:86-92.

19. Rasmussen BK, Olesen J. Migraine with aura and migraine without aura: an epidemiological study. Cephalalgia 1992;12:221-8; discussion 186.

20. Launer LJ, Terwindt GM, Ferrari MD. The prevalence and characteristics of migraine in a population-based cohort: the GEM study. Neurology 1999;53:537-42.

21. Kelman L. The triggers or precipitants of the acute migraine attack. Cephalalgia 2007;27:394-402.

22. Goadsby PJ, Lipton RB, Ferrari MD. Migraine - current understanding and treatment. N Engl J Med 2002;346: 257-70

23. Silberstein SD, Rosenberg J. Multispecialty consensus on diagnosis and treatment of headache. Neurology 2000;54:1553.

24. Silberstein SD, Holland S, Freitag F, et al. Evidencebased guideline update: pharmacologic treatment for episodic migraine prevention in adults: report of the Quality Standards Subcommittee of the American Academy of Neurology and the American Headache Society. Neurology 2012;78:1337-45.

25. Kelley NE, Tepper DE. Rescue therapy for acute mi- graine, part 3: opioids, NSAIDs, steroids, and post-discharge medications. Headache 2012;52:467-82.

26. Loder E, Rizzoli P. Tension-type headache. BMJ 2008;336:88-92.

27. Stovener LJ, Hagen K, Jensen R, et al. The global burden of headache: a documentation of headache prevalence and disability worldwide. Cephalalgia 2007;27: 193-210.

28. Laurell K, Larsson B, Eeg-Olofsson O. Prevalence of headache in Swedish schoolchildren, with a focus on tension-type headache. Cephalalgia 2004;24:380-8.

29. Scher AI, Stewart WF, Liberman J, Lipton RB. Prevalence of frequent headache in a population sample. Headache 1998;38:497-506.

30. Schwartz BS, Stewart WF, Simon D, Lipton RB. Epidemiology of tension-type headache. JAMA 1998;279: 381-3.

31. Ashina M. Neurobiology of chronic tension-type headache. Cephalalgia 2004;24:161-72.

32. Bendtsen L, Jensen R. Tension-type headache. Neurol Clin 2009;27:525-35.

33. Jensen R, Olesen J. Initiating mechanisms of experimentally induced tension-type headache. Cephalalgia 1996; 16:175-82.

34. Scottish Intercollegiate Guidelines Network (SIGN). 107: Diagnosis and management of headache in adults. A national clinical guideline; November 2008. Available from: http://www.sign.ac.uk/pdf/sign107.pdf

35. Lambru C, Matharu N. Trigeminal autonomic cephalalgias: a review of recent diagnostic, therapeutic and pathophysiological developments. Ann Indian Acad Neurol 2012;15:s51-61.

36. Katsarava Z, Obermann M, Yoon MS, et al. Prevalence of cluster headache in a population-based sample in Germany. Cephalalgia 2007;27:1014-9.

37. Bahra A, May A, Goadsby P. Cluster headache: a prospective clinical study with diagnostic implications. Neurology 2002;58:354-61.

38. Favoni V, Grimaldi D, Pierangeli G, et al. SUNCT/SUNA and neurovascular compression: New cases and critical literature review. Cephalalgia 2013;33:1337-48.

39. Wang SJ, Fuh JL. The "other" headaches: primary cough, exertion, sex, and primary stabbing headaches. Curr Pain Headache Rep 2010;14:41-6.

40. Bahra A. Other primary headaches. Ann Indian Acad Neurol 2012;15:S66-71.

41. Abrams BM. Medication overuse headaches. Med Clin North Am 2013;97:337-52.

42. Mortimer AM, Bradley MD, Stoodley NG, Renowden SA. Thunderclap haedache: diagnostic considerations and neuroimaging features. Clin Radiol 2012;XXX:e1-13.

43. Ducros A, Bousser MG. Thunderclap headache. BMJ 2012;345:e8557.

44. Linn FH, Rinkel GJ, Algra A, van Gijn J. Headache characteristics in subarachnoid haemorrhage and benign thunderclap headache. J Neurol Neurosurg Psychiatry 1998;65:791-3.

45. Edlow JA, Caplan LR. Avoiding pitfalls in the diagnosis of subarachnoid hemorrhage. N Engl J Med 2000;342: 29-36.

46. Landtblom AM, Fridriksson S, Boivie J, et al. Sudden onset headache: a prospective study of features, incidence and causes. Cephalalgia 2002;22:354e60. 
47. Savitz SI, Edlow J. Thunderclap headache with normal $\mathrm{CT}$ and lumbar puncture: further investigations are unnecessary. Stroke 2008;39:1392-3.

48. AGREE Next Steps Consortium. Appraisal of Guidelines for Research \& Evaluation II - AGREE II Instrument; May 2009. Available from: http://www.agreetrust.org/wpcontent/uploads/2013/10/AGREE-II-Users-Manual-and23-item-Instrument_2009_UPDATE_2013.pdf

49. Pringsheim T, Davenport WJ, Mackie G, et al. Canadian Headache Society guideline for migraine prophylaxis. Can J Neurol Sci 2012;39:S1-S2.

50. Toward Optimized Practice. Guideline for primary care management of headache in adults. Edmonton (AB): Toward Optimized Practice; 2012. Available from: http://www.guideline.gov/content.aspx?id=47060

51. Ramadan NM, Silberstein SD, Freitag FG, et al. Evidence-based guidelines for migraine headache in the pri- mary care setting: pharmacological management for prevention of migraine. Available from: http://tools.aan. com/professionals/practice/pdfs/g10090.pdf

52. National Clinical Guideline Centre. Headaches: diagnosis and management of headaches in young people and adults. London: National Institute for Health and Clinical Excellence (NICE); 2012. Available from: http:// www.guideline.gov/content.aspx? $\mathrm{id}=38444$

53. National Institute for Health and Clinical Excellence (NICE). Headaches: diagnosis and management of headaches in young people and adults. NICE guideline [CG150]; September 2012. Available from: http://www. nice.org.uk/guidance/cg150/chapter/guidance

54. Maya A, Leoneb M, Áfrac J, et al. Linee Guida EFNS per il trattamento della cefalea a grappolo e delle altre cefalee autonomico-trigeminali. EFNS Eur J Neurol 2006;13:1066-77. 